FORMATION Formation emploi

Revue française de sciences sociales

108 | octobre-décembre 2009

Les processus de professionnalisation

\title{
Le cadre européen des certifications : quelles stratégies nationales d'adaptation?
}

The european qualifications framework: what national strategies of adjustment? Der europäische Rahmen für Zertifizierungen: welche nationale Anpassungsstrategien gibt es?

El marco europeo de las certificaciones : ¿que estrategias nacionales de adaptacion?

\section{Philippe Méhaut et Christopher Winch}

\section{OpenEdition}

Journals

Édition électronique

URL : http://journals.openedition.org/formationemploi/2115

DOI : 10.4000/formationemploi.2115

ISSN : 2107-0946

\section{Éditeur}

La Documentation française

\section{Édition imprimée}

Date de publication : 1 décembre 2009

Pagination : $97-111$

ISSN : 0759-6340

Référence électronique

Philippe Méhaut et Christopher Winch, « Le cadre européen des certifications : quelles stratégies

nationales d'adaptation? », Formation emploi [En ligne], 108 | octobre-décembre 2009, mis en ligne le 01 janvier 2012, consulté le 30 octobre 2020. URL : http://journals.openedition.org/formationemploi/ 2115 ; DOI : https://doi.org/10.4000/formationemploi.2115 


\section{Comparaison européenne}

\section{Le cadre européen}

\section{des certifications : quelles stratégies nationales d'adaptation?}

Philippe Méhaut et Christopher Winch*

Le cadre européen des certifications interpelle les systèmes nationaux de diplômes. L'examen des structures de certifications de quatre pays révèle une certaine diversité et des conceptions parfois difficilement compatibles. Dès lors, quelques hypothèses de stratégies nationales de transposition sont formulées.

Les comparaisons internationales sur les processus de construction et les formes des certifications se sont développées. L'un des travaux fondateurs est l'ouvrage de Möbus et Verdier (1997) sur la comparaison France-Allemagne. En France, les National Vocational Qualifications (NVQs) britanniques ont fait l'objet d'une attention particulière dans la mesure où elles représentaient $a$ priori l'antithèse du système français (Bessy, 2000). L'une des conclusions fréquente de ces travaux est d'insister sur l'importance des différences sociétales qui marquent les systèmes de certification, comme d'ailleurs les systèmes de formation professionnelle (Bosch, Charest, 2009).

Pour autant, notamment depuis l'agenda de Lisbonne, des politiques européennes plus actives se développent. La formation professionnelle rentre dans le champ de la méthode ouverte de coordination (Laffan, Shaw, 2005). D'autres outils de coordination se développent, dont le cadre européen de certification (CEC) et les futurs crédits européens de formation professionnelle (ECVET).

Les effets cumulés de ces diverses impulsions européennes ne doivent pas être négligés. Ils sont susceptibles d'influer les trajectoires nationales. Ceci est particulièrement sensible dans les pays nouveaux entrants dans l'Union européenne, assez prompts à adopter toutes ces initiatives, d'autant que celles-ci

Philippe Méhaut est directeur de recherche CNRS au LEST (Laboratoire d'économie et de sociologie du travaill), Aix-en-Provence. Il a été directeur adjoint du Céreq. Ses travaux portent sur les politiques et pratiques de formation et sur la dynamique des marchés du travail. II a contribué à I'ouvrage Low Wage Work in Wealthy Countries (Gautié et Schmitt Eds.), Russell Sage, New York, (2009) ainsi qu'à l'ouvrage (à paraitre) dont est issu cet article, Knowledge, Skills, Competence: towards equivalence of European vocational qualifications? Brockmann, Clarke, Winch (Eds.) Taylor and Francis, Londres, (2010). 
Christopher Winch est professeur de philosophie de l'éducation et chef du département d'éducation et d'études professionnelles au King's Collège de Londres. Ses travaux principaux portent sur la philosophie de l'éducation et de la formation professionnelle. Il a récemment publié : "Gilbert ryle and the possibility of vocational education » in Journal of applied philosophy; un livre, Dimensions of expertise, sera publié par Continuum books début 2010.

ouvrent alors souvent droit à des financements européens. Bien que fondé sur le pur volontariat, se présentant comme un outil souple pour la construction d'une confiance mutuelle, le CEC peut influencer, à terme, la conception et la structure des certifications nationales.

L'objectif de cet article est de lire, à l'aune des principes du CEC, différents systèmes nationaux de certification. Dans une première partie, on propose une analyse des fondements et objectifs du CEC. Une deuxième partie, basée sur des études de diplômes dans quatre pays (Grande Bretagne, France, Pays Bas, Allemagne) confronte certains des fondements conceptuels et institutionnels de ces diplômes à ceux du cadre européen. Une troisième partie propose ensuite quelques scénarios sur la future articulation entre cadres nationaux et européen. Cette analyse se base principalement sur un travail comparatif réalisé pour la fondation Nuffield (Brockmann, Clarke, Winch 2009, pour une synthèse des travaux).

\section{LE CADRE EUROPÉEN DES CERTIFICATIONS : UN NOUVEL OUTIL DE TRANSPARENCE ET DE COORDINATION?}

\begin{abstract}
Adopté par le parlement européen en 2008, le Cadre européen des certifications (CEC) se présente comme un "méta-cadre », censé créer à la fois un langage commun et des références communes entre les certifications des différents pays membres de l'Union européenne. Il s'inscrit dans la tendance générale, soutenue par les organisations internatio-
\end{abstract}

\section{Encadrél \\ Étapes et outils de la politique européenne en matière de formation}

Le sommet européen de Lisbonne (2000), en traçant comme perspective une Europe compétitive dans la société de la connaissance, a donné une nouvelle impulsion à la politique européenne de formation professionnelle, confirmée depuis par les déclarations de Copenhague et plus récemment de Bordeaux (2008). Bien que ne faisant pas partie des compétences de l'Union, l'éducation et, à un degré plus fort, la formation professionnelle, rentrent néanmoins progressivement dans des processus d'énoncé et de coordination de politiques à l'échelle de l'Union.

Ainsi la formation professionnelle est rentrée progressivement dans le cadre de la méthode ouverte de coordination : choix volontaire des États de se saisir d'un sujet au niveau communautaire ; fixation de lignes politiques, d'objectifs et d'indicateurs; puis stratégies nationales pour atteindre ces objectifs.

À côté de grands programmes bien connus, comme Erasmus et Léonardo, le Fonds social européen abonde des politiques nationales ou régionales (par exemple de développement de l'apprentissage).

Enfin des outils cadres de références sont proposés pour favoriser l'harmonisation des systèmes. Outre le Cadre européen de certification (CEC), on peut mentionner la construction en cours d'un système de crédits européens (ECVET) permettant de modulariser des unités de formation et de faciliter la mobilité internationale des élèves et étudiants. Ce système s'inspire, pour la formation professionnelle, du système de crédits européens mis en place pour l'Université dans le cadre du processus dit "de Bologne », qui veut à la fois harmoniser les niveaux de formation (le «L-M-D» - licence-maitrise-doctorat) et faciliter la mobilité des étudiants. 
nales (notamment l'OCDE - Organisation de coopération et de développement économiques), à développer de tels cadres aux niveaux national et transnational. Les objectifs généralement affichés sont ceux d'une plus grande transparence des certifications, d'une « mise en ordre », notamment lorsque celles-ci sont plurielles et nombreuses.

Si le CEC n'échappe pas à ces objectifs, il semble cependant, parfois explicitement, parfois implicitement, en poursuivre aussi d'autres ( $c f$. notamment Sellin, 2008), d'où d'inévitables flous dans les concepts, et d'éventuelles tensions entre des objectifs très nombreux et parfois contradictoires.

- Le CEC se présente d'abord comme un cadre englobant ayant vocation à dépasser les frontières entre formation professionnelle et éducation générale. Il couvre ainsi en principe l'enseignement supérieur. En ce sens, il s'inspire et suit le processus de Bologne (les licences professionnelles, les masters et les doctorats pourraient/devraient y trouver place). Ce dépassement traduit le mouvement engagé dans la plupart des pays européens vers l'élévation des niveaux de formation, vers la poursuite d'études dans un enseignement supérieur qui se professionnalise. Mais il traduit aussi l'un des axes souvent réaffirmé des politiques européennes, celui de la «parité d'estime » entre enseignement général et formation professionnelle.

- Le CEC s'inscrit ensuite pleinement dans la politique européenne de la formation tout au long de la vie. Il entend donc couvrir sans distinction les certifications en "formation initiale» et en "formation continue » (pour prendre la terminologie française, même si nous savons que cette distinction n'a pas nécessairement sens dans d'autres pays (Verdier, 2008 ; voir aussi Morvan, 2006)). Mais il va plus loin puisqu'il vise, suivant en cela la terminologie et les recommandations européennes, des certifications qui pourraient sanctionner des acquisitions via l'éducation «formelle », « informelle » et « non formelle $»^{1}$.

- Pour ce faire, mais aussi parce qu'il a enregistré l'échec de la plupart des tentatives européennes antérieures de construction d'équivalence entre cer-

\footnotetext{
${ }^{1}$ L'homologue français de cette trilogie est approximativement les formations diplômantes/certifiantes, les formations « formelles » mais non certifiantes et les formations informelles (apprentissage sur le tas, acquis de l'expérience).
}

tifications nationales ( $c f$. par exemple Bouder, Kirsch, 2008, pour un très bon historique), il se fonde sur les «learning outcomes» (Jessup, 1991) que l'on pourrait traduire en français par «acquis de l'apprentissage ». Il entend donc rompre avec les approches par les «inputs-entrants» (nombres d'années d'études, nombre d'heures de formation) et avec celles en termes «d'output-produit» (par exemple une moyenne à un examen attestant d'un niveau de connaissances). De façon explicite, dans le glossaire qui appuie le CEC, les «learning outcomes» (acquis de l'apprentissage) qui sont prouvés dans un contexte spécifique » (ce qui serait, dit le glossaire, le cas des situations d'apprentissage professionnel - mais pourquoi seulement de celles là ?) sont équivalents aux compétences ». Le CEC s'inscrit alors plutôt dans la tradition anglo-saxonne (Oates, 2004). Il suit les pressions exercées, notamment par l'OCDE, en faveur d'un «pilotage par l'aval » des systèmes de formation (Vinokur, 1995), tendant à renverser leurs processus de construction, mais aussi le poids des acteurs au sein de ces processus (Bjornavold, Coles, 2008). L'une des implications possibles, à terme, est celle de l'évolution des logiques de financement des établissements, qui prendraient plus appui sur les output et sur les outcomes ( $c f$. Felstead, 1998, pour un panorama de cette question).

- Outre un cadre d'analyse de ces acquis de l'apprentissage (learning outcomes) le CEC entend proposer une grille harmonisée de leurs niveaux. Ici aussi on peut noter plusieurs objectifs. Le premier est celui de la lisibilité «horizontale» des niveaux de certification entre pays. Cette harmonisation est censée être nécessaire dans une logique de mobilité européenne des élèves et étudiants. En ce sens notamment, le CEC est présenté comme un "préalable» aux ECVET, comme les descripteurs de Bologne l'ont été aux ECTS $^{2}$. Le deuxième objectif est celui de la lisibilité verticale. Ici on se situe plutôt en intra-national, dans la perspective de la formation tout au long de la vie et

\footnotetext{
2 Pour attribuer des « crédits » permettant de valider ailleurs une unité de formation faite dans un pays, encore faut-il que les formations soient en correspondance. Pour l'université, c'est l'architecture dite «L-M-D » licence, master, doctorat qui permet de situer les différentes unités/modules de formation et les crédits qui leur correspondent. La même logique, appliquée à la formation professionnelle, suppose que les diplômes visés soient articulés entre eux dans une grille de référence.
} 
des passerelles verticales d'une certification à l'autre (comme dans la logique des niveaux à la française ou à la hollandaise). Mais un troisième objectif, plus implicite celui-là, est celui des niveaux « sur le marché du travail ». Le CEC est en effet aussi présenté comme un outil de mobilité des travailleurs, visant à faciliter non seulement le positionnement des certifications/diplômes, mais aussi celui des qualifications (ici au sens français du terme ${ }^{3}$ ). L'examen des learning outcomes révèle cette ambiguïté. Les « compétences » sont ainsi formulées autour de la

3 «Qualification» au sens anglais du terme désigne un titre, un diplôme. Le «European Qualification Framework» a été traduit en français par " cadre européen des certifications » pour éviter l'ambiguïté avec le mot français de qualification qui renvoie lui aux caractéristiques du poste de travail et/ou de l'individu. notion d'autonomie qui renvoie à la fois à celle d'autonomie dans les apprentissages, mais aussi à celle d'autonomie sur le poste de travail. Markowitsch (2008, p. 60) souligne bien ce qu'il appelle les trois attributs du CEC que sont la hiérarchie des tâches et des fonctions, celle des systèmes éducatifs et celle de l'acquisition des compétences. En ce sens, le CEC n'échappe pas totalement aux exercices antérieurs, dont certains partaient de l'analyse du travail.

On le voit, la notion de "méta-cadre » peut dès lors être interprétée sous un triple sens. Méta au sens de cadre auquel peuvent s'articuler les différents cadres nationaux de certification (ce qui suppose une certaine plasticité, une possibilité d'appropriation de son langage). Méta au sens où il se donne vocation d'absorber toutes les certifications, quelles que soient

\section{Encadré 2}

\section{Méthodologie}

Les analyses développées ci-dessous s'appuient sur un travail comparatif réalisé pour la Fondation Nuffield (*). Dans une première étape, les principales caractéristiques des systèmes de certification et de formation professionnelles de quatre pays (Hollande, Allemagne, France, Royaume Uni, en fait principalement l'Angleterre) ont été analysées. Dans une seconde étape, quatre certifications ont été analysées finement. Dans chaque cas, on a procédé à des interviews des principaux acteurs lau sein des commissions professionnelles consultatives ou de leurs équivalents pour la France, des structures de pilotage dans les autres pays). Entre quatre et huit entretiens approfondis ont été menés, sur une grille commune qui balayait les principales dimensions des certifications : conception de la (des) compétence(s), rapports aux savoirs et à l'éducation, gouvernance, rapport au marché du travail. Ont été aussi compilés et analysés les principaux documents supports de ces certifications (référentiels ou leurs homologues). Un rapport national par pays a été réalisé sur la base de ces investigations, puis une analyse de synthèse comparative, appuyée notamment par trois séminaires entre les chercheurs impliqués. Les certifications choisies l'ont été pour la variété des situations qu'elles recouvraient (industrie/tertiaire, niveaux au sens français du terme...). Tout en ayant conscience que les cibles « emploi/métier » visées par ces certifications varient d'un pays à l'autre du fait des différences dans la division et l'organisation du travail, nous avons pris le parti de comparer ces certifications, moins sur leur contenu (par exemple sur les programmes de formation, le contenu des compétences) que sur les concepts et les processus de conception qui les sous-tendent. Ont ainsi été étudiées les certifications de maçon (CAP maçon pour la France), de conducteur de poids lourd (titre ministère du Travail pour la France), d'informaticien (DUT - diplôme universitaire de technologie) et d'infirmier (diplôme d'Éłat). Ce choix présente l'avantage de couvrir une variété de niveaux (pour les pays où une organisation en niveaux existe et, pour la France, du niveau V - CAP / BEP - au niveau III - BTS/DUT) et une pluralité d'organismes certificateurs.

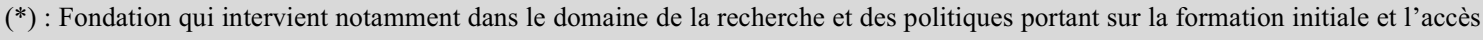
au marché du travail des jeunes. 
leur nature, leurs finalités, leurs origines. Méta au sens où, tout en affirmant que sa finalité première est d'abord celle des politiques d'éducation et de formation, il déborde de fait celles-ci pour aller vers l'antienne de la « libre circulation des travailleurs».

Il est dès lors relativement inévitable que les notions sur lequel il se fonde soient floues. Instrument de "politique pragmatique» (Markowitsch, 2008, p. 61), il est marqué par le «manque de fondements théoriques ou de base systématique » (ibid).

Or ce méta-cadre doit être saisi par des acteurs dans des contextes nationaux différents. Les analyses comparatives sur les certifications (voir par exemple Möbus et Verdier, 1997), celles sur les systèmes nationaux de formation professionnelle (Greinert, 2005; Gehin, Méhaut, 1993 ; Clarke et Winch, 2007 ; Bosch, Charest, 2010) soulignent les différences institutionnelles, dans les mécanismes de régulation, dans la construction des certifications, dans leur rapport au marché du travail. Il nous faut donc confronter les différentes formes de construction des certifications aux notions du CEC, avant d'en venir ensuite à la question de la transposition des cadres nationaux dans ce méta-cadre européen.

\section{LES CERTIFICATIONS NATIONALES À L'AUNE DES CONCEPTS DU CADRE EUROPÉEN DES CERTIFICATIONS}

Nous reviendrons d'abord rapidement sur quelques caractéristiques institutionnelles majeures des systèmes nationaux de formation et de certifications professionnelles, avant d'aborder les concepts fondamentaux qui sous-tendent ces certifications.

\section{Des systèmes nationaux pluriels}

L'analyse comparative des systèmes de formation professionnelle met en général l'accent sur la cohérence nationale et les différences systémiques entre pays. Les variables couramment retenues sont celles du mode de financement, du contrôle par les acteurs, du mode d'organisation de la formation, du rapport au marché du travail... Les travaux qui partent du système de certification et de la construction des diplômes sont plus rares. Mais ils insistent aussi sur les différences dans les configurations d'acteurs, dans la valeur relative des certifications, dans la structure des référentiels ( $c f$. Mobus et Verdier, 1997, pour une comparaison France/Allemagne).

Il ne s'agit pas ici, à partir de l'étude de quatre diplômes, de reprendre ces comparaisons systémiques. On se contentera de souligner quelques caractéristiques induites de la structure et du mode de construction des certifications.

La Hollande présente la situation nationale la plus homogène : un cadre de certification unique pour nos quatre diplômes, un même principe de construction avec une forte implication des partenaires sociaux, une conception étendue des compétences, deux voies de formation (scolaire et apprentissage, cette dernière plutôt en régression) (Westerhuis, 2009).

Les proximités avec la France sont assez fortes, au moins pour ce qui est des diplômes relevant en France de l'Éducation nationale. Toutefois, en France, la place des partenaires sociaux est plus faible et toujours en tension. Les quatre diplômes relèvent ici, par choix d'échantillon, de quatre autorités différentes (Travail, Éducation, Santé et Enseignement supérieur). Comme en Hollande, certains sont accessibles par la voie scolaire et par l'apprentissage. Ceci permet de souligner une certaine hétérogénéité interne du système français, parfois sousestimée dans les comparaisons macro (Méhaut, 2009). Comme le souligne Verdier (2008), différents « régimes » peuvent coexister et s'hybrider au sein d'un même système.

C'est aussi le cas en Allemagne. Si deux diplômes (maçon et conducteur) relèvent du classique système dual ( $c f$. Bosch, 2010 pour une analyse récente et en dynamique du système allemand), celui d'infirmière y échappe (écoles de formation). Il en va de même de celui d'informatique (il s'agit toutefois ici du choix d'une certification "atypique », mais en fort développement ; d'autres certifications dans le même domaine s'inscrivent dans le système dual). Là aussi, on note une certaine hétérogénéité des certifications, semble-t-il croissante dans la mesure où le pourcentage d'une classe d'âge passant par le système dual 
diminue. Néanmoins, le concept de base de «beruf » (métier) demeure un socle commun (Hanf, 2009).

L'Angleterre présente la situation la plus éclatée. Dans deux cas (maçon et conducteur), on est de façon dominante sous le régime des $N V Q s$. Le diplôme d'infirmière est régi par un autre système et intégré à l'université. Plusieurs voies et titres coexistent, comme en France, pour l'informatique, avec des conceptions assez différentes : apprentissage, formation professionnelle formelle, formation professionnelle universitaire ; c'est le diplôme de cette voie qui est plus particulièrement analysé (Brockmann et alii, 2009).

Les différences structurelles entre pays sont souvent avancées comme argument rendant difficile la mise en œuvre d'un cadre européen unique. On voit cependant ici qu'il existe aussi des différences internes à chaque pays, ce qui lève pour partie cet argument. De fait, seul les titres $N V Q s$, conçus comme totalement séparés de la formation elle- même, se distinguent assez nettement de ce que l'on observe dans les autres pays et pour les autres titres où les proximités sont plus fortes. La quasi-absence des organisations syndicales et, à l'exception du diplôme d'infirmière, la quasi-absence de couplage « institutionnel» (conventions collectives) avec le marché du travail opposent cependant aussi nos cas anglais aux autres cas continentaux.

\section{Des conceptions de la compétence hétérogènes}

Le CEC est basé sur un "modèle de la compétence » qui se décline en trois dimensions, les savoirs, les aptitudes et, d'une façon qui peut troubler, les compétences ( $c f$. tableau 1). Cette notion plus restreinte que la notion «chapeau » recouvre alors principalement le degré d'autonomie et de responsabilité (au poste de travail ou dans un processus d'apprentissage).

Tableau 1

Le principe du Cadre européen des certifications (CEC)

\begin{tabular}{|c|c|c|c|}
\hline $\begin{array}{l}\text { Niveau/ } \\
\text { Compétences }\end{array}$ & $\begin{array}{l}\text { Savoirs (théoriques } \\
\text { ou factuels) }\end{array}$ & $\begin{array}{l}\text { Aptitudes (cognitives } \\
\text { ou pratiques) }\end{array}$ & $\begin{array}{c}\text { Compétences (responsabilité } \\
\text { ou autonomie }\end{array}$ \\
\hline \multicolumn{4}{|l|}{8 (le plus haut) } \\
\hline \multicolumn{4}{|l|}{7} \\
\hline \multicolumn{4}{|l|}{6} \\
\hline 5 & $\begin{array}{l}\text { Savoirs détaillés, } \\
\text { spécialisés, factuels et } \\
\text { théoriques dans un domaine } \\
\text { de travail ou d'études, } \\
\text { et conscience des limites } \\
\text { de ces savoirs }\end{array}$ & $\begin{array}{l}\text { Gamme étendue d'aptitudes } \\
\text { cognitives et pratiques } \\
\text { requises pour imaginer } \\
\text { des solutions créatives } \\
\text { à des problèmes abstraits }\end{array}$ & $\begin{array}{l}\text { Gérer et superviser dans des contextes } \\
\text { d'activités professionnelles ou d'études } \\
\text { où les changements sont imprévisibles } \\
\text { Réviser et développer ses performances } \\
\text { et celles des autres }\end{array}$ \\
\hline 4 & $\begin{array}{l}\text { Savoirs (théoriques ou } \\
\text { factuels) dans des contextes } \\
\text { généraux dans un domaine } \\
\text { de travail ou d'études }\end{array}$ & $\begin{array}{l}\text { Gamme d'aptitudes requises } \\
\text { pour imaginer des solutions } \\
\text { à des problèmes précis }\end{array}$ & $\begin{array}{l}\text { S'autogérer dans la limite des consignes } \\
\text { dans des contextes généralement } \\
\text { prévisibles mais susceptibles de } \\
\text { changer. } \\
\text { Superviser le travail habituel d'autres } \\
\text { personnes... Responsabilité pour l'éva- } \\
\text { luation et l'amélioration des activités }\end{array}$ \\
\hline \multicolumn{4}{|r|}{ 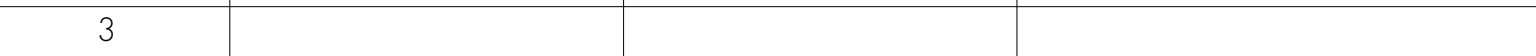 } \\
\hline \multicolumn{4}{|l|}{2} \\
\hline 1 (le plus bas) & & & \\
\hline
\end{tabular}

Lecture : toute certification devrait trouver sa place dans le cadre européen à partir du niveau de maitrise des savoirs, aptitudes et compétences auxquels elle prépare. 
C'est cette notion clé (compétence au sens large et sa déclinaison) qui doit en principe permettre de situer dans le CEC tout titre ou diplôme sur la base des acquis de l'apprentissage, indépendamment des voies d'acquisition de cette (ces) compétence (s). Il est donc fondamental que cette notion soit comprise de la même façon dans les différents pays.

Les trois pays continentaux utilisent un concept multidimensionnel de compétence, recouvrant l'intégration de savoirs et savoir-faire dans la pratique ainsi que des aptitudes comportementales qui sont pensées en général autour de l'unité d'un métier (ou d'une famille de métiers) plutôt qu'autour de tâches séparées. L'articulation entre les trois dimensions de la compétence au sens du CEC y est forte.

En Angleterre, tout particulièrement pour les $N V Q s$, la notion de compétence est beaucoup plus étroite. Elle se fonde sur une performance au poste de travail, correspondant plutôt à un ensemble de tâches élémentaires, non nécessairement articulées à une notion de métier. Cette conception, qui renvoie à la notion clé de «skills», fait davantage référence à des aptitudes comportementales prouvées en situation de travail qu'à la maitrise sous-jacentes de "compétencessavoir » au sens continental du terme.

Les « attitudes » sont un élément explicite du système hollandais, correspondant peu ou prou au «savoir être » français et aux compétences (Kompetenzen) sociales et personnelles allemandes. Toutefois, en Hollande, la dimension civique et morale citoyenne - de ces «attitudes » est plus fortement marquée que dans les deux autres pays. En Angleterre, l'accent est fortement mis sur les «soft skills ${ }^{4}$, mais qui n'ont pas la même étendue et la même signification que les attitudes ou savoir être. Cette conception étroite est particulièrement significative pour le maçon et le conducteur : dans les deux cas, l'accent est mis sur la capacité à « exécuter ce qui est prescrit». La compétence personnelle est alors l'aptitude à mettre en œuvre ces « skills » en référence au standard prédéfini.

\footnotetext{
${ }^{4}$ Les compétences dites douces sont orientées vers les interactions humaines et font appel à l'intelligence émotionnelle (communication, négociation, empathie, gestion des conflits, du temps, etc.). Elles sont complémentaires à la formation académique ou technique. Mais pour certains auteurs, les soft skills joueraient un rôle croissant sur le marché du travail.
}

De fortes différences existent aussi, essentiellement pour le maçon et le conducteur dans la notion et le rapport aux «savoirs». En France, comme en Hollande mais aussi en Allemagne, les référentiels incluent explicitement une gamme de savoirs théoriques et pratiques. De plus, dans les deux premiers pays, la gradation en niveaux du système de formation professionnelle et les possibilités de passage de l'un à l'autre supposent une maîtrise graduée de différentes gammes de savoirs, alors que les $N V Q s$ ne comportent pas de référence explicite à cette maîtrise.

En revanche, la référence aux savoir théoriques est présente dans tous les pays pour les deux autres diplômes (informatique et infirmier).

Si l'on schématisait, on pourrait ainsi dire qu'en général le «grain » de compétence est plus petit au Royaume-Uni que dans les trois autres pays. Et particulièrement lorsque l'on regarde les $N V Q s$, ces grains sont séparés, on ne considère pas ou peu l'épi... alors que c'est l'épi qui prime dans les trois autres pays. La conception de la compétence y est plus « utilitariste», renvoyant plus à la performance dans l'accomplissement d'une tâche. De même, l'articulation entre les savoir-faire et le substrat de connaissances (théoriques, pratiques) qui permettent leur mise en œuvre, est rarement explicite.

Toutefois, cette première lecture qui met l'accent sur «l'idéal type » de compétence dans chaque pays, et qui conduirait à opposer le modèle du Royaume-Uni à un modèle «continental» masque, comme nous l'avons dit, des différences/nuances internes aux pays selon le type de certification examinée.

Le graphique 1 positionne les certifications étudiées de chaque pays sur les deux axes que sont d'une part la plus ou moins grande intégration des savoirs dans la conception de la compétence et d'autre part selon le caractère plus ou moins intégré de celle-ci.

Si les certifications des trois pays continentaux sont plutôt groupées dans le quart Nord Ouest, des exceptions apparaissent. Ainsi, le certificat d'informatique Allemand (qui, rappelons-le, est en dehors du système dual) est moins basé sur des savoirs que sur des savoir-faire, savoir-être (pour prendre ici une terminologie française). Le titre français de conducteur (qui 
Graphique 1

Conception des compétences selon les différents diplômes

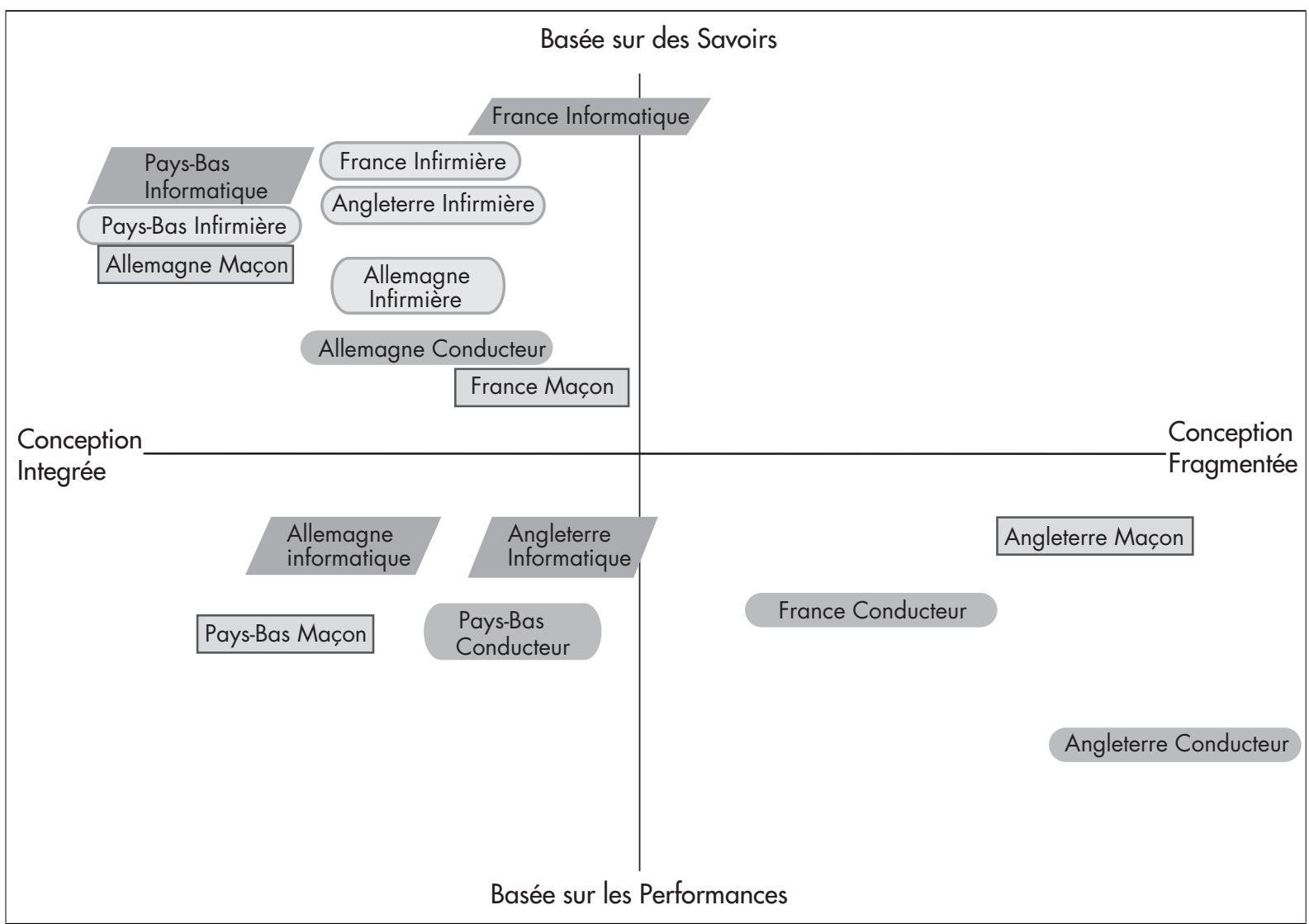

Note de lecture : Le graphique 1 positionne les certifications étudiées de chaque pays sur les deux axes que sont d'une part la plus ou moins grande intégration des savoirs dans la conception de la compétence et d'autre part selon le caractère plus ou moins intégré de celle-ci.

Source : Brockmann et al, 2009.

relève, rappelons-le, de la " formation continue ») se rapproche de ses homologues hollandais et anglais. À l'inverse, la formation des infirmières anglaises échappe aux dominantes nationales pour se rapprocher fortement des certifications continentales.

\section{La logique des « learning outcomes » interroge le rapport certification/éducation}

Le CEC se présente résolument comme basé sur une logique des learning outcomes (acquis de l'apprentissage). Le classement des certifications est donc en principe indépendant des modalités d'acquisition des compétences. Le niveau des compétences attesté par la certification est ainsi indépendant par exemple d'une durée de formation. Selon les pays et les certifications étudiées, cette logique est plus ou moins présente.

En Hollande, tout le système (et donc les quatre certifications) est organisé autour d'une logique d'outcomes. Pour prendre un terme français, un référentiel de compétences est à la base de la certification. À charge aux opérateurs de formation de définir les voies par lesquelles ces compétences sont atteintes. Néanmoins, tant dans la formulation de ces compétences que dans l'organisation du système de formation lui-même, on peut difficilement séparer totalement les outcomes des inputs (autrement dit les « compétences» produites et la façon de les produire). Le lien certification/formation demeure fort (Westerhuis, 2009). 
La France présente un paysage plus éclaté. Certes, et depuis longtemps, l'essentiel des diplômes professionnels de l'Éducation nationale ainsi que les titres ministère du Travail sont bâtis autour du couple référentiel d'activité/référentiel de certification (cf. Maillard, 2008). C'est ce dernier, souvent énoncé en termes de " capacités/compétences », qui permet en principe d'évaluer les compétences indépendamment de leur mode d'acquisition. En ce sens, ils sont aussi régis par la logique des learning outcomes. Néanmoins, l'examen du CAP maçon (mais aussi d'autres diplômes) montre que les frontières sont poreuses et parfois difficiles à définir entre les deux référentiels (activité/certification, mais aussi entre ces deux référentiels et le processus de formation). D'une part, on trouve souvent une rubrique dite de « savoirs associés » qui vient s'articuler aux capacités/compétences; d'autre part, comme en Hollande, la traduction en programmes de formation est inséparable du processus d'ensemble.

Au moment de notre recherche de terrain, le diplôme d'État d'infirmier était encore régi par une logique d'input. Le référentiel de compétence était en cours de construction. Et, dans ce domaine d'un métier régulé, il y a à l'évidence tension entre la pure logique d'outcomes du CEC et la régulation par les inputs (nombre d'heures de formation) prévue par la directive européenne qui organise la reconnaissance mutuelle de ce diplôme. À l'image du diplôme infirmier, le DUT (diplôme universitaire de technologie) informatique est essentiellement bâti autour d'une logique d'input - programme national, nombre d'heures par bloc de matières... C'est le titre ministère du Travail de conducteur de poids lourds qui, dans sa structure et sa conception, se rapproche le plus des notions du CEC. C'est aussi celui qui est le plus «atypique » dans le paysage français, et qui présente le plus d'analogies avec la $N V Q$ britannique. Mais il faut tenir compte ici du fait qu'il s'agit d'un titre «formation continue $»^{5}$ (Méhaut, 2009).

\footnotetext{
${ }^{5}$ Il existe aussi une réglementation européenne prévoyant un certificat d'aptitude complémentaire au permis de conduire, le certificat «Fimo». Ce certificat peut être délivré, soit sur la base d'un module de formation avec horaire prédéfini (donc plutôt dans une logique d'input), soit sur la base d'une preuve de ces aptitudes (donc plutôt dans une logique d'outcomes). De nos quatre pays, seule l'Angleterre a fait le deuxième choix.
}

En Allemagne, l'essentiel du système dual est certes régi par la définition de cibles «métier», donc pourrait être considéré aussi comme répondant à une logique d'outcomes. Néanmoins, le cœur du système reste basé sur une logique d'inputs, que l'on retrouve dans trois certifications. Seul ici le diplôme d'informatique, à l'inverse de la France, échappe à cette logique et serait le plus proche d'une construction par les outcomes (Hanf, 2009).

En Angleterre, le système est aussi de type mixte. Le diplôme d'infirmier est toujours basé sur une logique d'inputs. Les formations d'informaticien sont aussi variées, avec plusieurs voies de formation correspondant à plusieurs certifications : le Higher Apprenticeship, principalement étudié ici, est composé de deux éléments principaux, une $N V Q$, logique d'outcomes, et le Foundation Degree, logique d'input. La formation de maçon comporte deux voies principales, une $N V Q$ et l'apprentissage traditionnel, mais le plus important facteur sur le marché du travail est l'expérience et l'apprentissage sur le tas. Pour le conducteur, il y a naturellement le permis de conduire ainsi qu'une $N V Q 2$, que moins de $10 \%$ des conducteurs possèdent et qui a une valeur très limitée sur le marché du travail. On peut dire que la formation des maçons en Angleterre diffère le plus de celle des trois autres pays. Il y a une faible relation entre l'industrie et les institutions éducatives ; la formation est basée principalement sur la pratique de chantier et est de type informel. Les outcomes sont des habiletés qui sont étroitement alignées sur la division traditionnelle du travail. L'organisation en unités séparées (qui peuvent durer de six semaines à deux ans) fait éclater l'unité de métier (Brockmann et alii, 2009).

\section{Une grille de niveaux qui touche à des enjeux plus ou moins forts selon les pays}

Le CEC propose, nous l'avons évoqué, une articulation entre des compétences (en colonne) et des niveaux de maîtrise de ces compétences (en ligne). La grille de niveaux en huit positions échelonne la maîtrise des « compétences » :

- de la «maitrise des savoirs généraux de base » (niveau 1) à la maîtrise de savoirs «à la frontière la plus avancée d'un domaine... » (niveau 8); 
- des « aptitudes de base pour effectuer des tâches simples » (niveau 1) aux « aptitudes et techniques les plus avancées et les plus spécialisées...» (niveau 8);

- de la compétence à «travailler ou étudier sous supervision dans un cadre structuré » (niveau 1) à celle de «démontrer un niveau élevé d'autorité, d'innovation, d'autonomie... » (niveau 8).

Cette organisation en niveaux doit en principe permettre de classer les certifications nationales, avec le double objectif d'équivalence horizontale et d'enchaînement vertical (Coles, Oates, 2004). Outre l'ambiguîté, déjà soulignée, des descripteurs de compétence valant à la fois pour le champ des études et pour celui du travail, cette grille introduit aussi des zones d'incertitude. D'une part, elle est articulée à celle du processus de Bologne. Les niveaux 5 à 8 sont explicitement référés aux différents niveaux des descripteurs de Bologne, le niveau 5 correspondant au premier cycle court de l'enseignement supérieur, le niveau 8 aux études doctorales. Ceci devrait entraîner, dans les pays où existe déjà une grille de niveaux, une transposition «descendante » dont nous allons voir qu'elle n'est pas simple. Par ailleurs, le niveau 1 de la grille peut être interprété de deux façons. Soit il s'agit de « compétences de base » qui correspondraient par exemple à la fin de scolarité obligatoire en France, soit il s'agit d'un premier niveau de formation professionnelle (par exemple le CAP).

Dans trois de nos pays, il y a des niveaux explicites tant pour l'enseignement général que pour la formation professionnelle.

- Dans deux pays (France et Pays Bas), ces niveaux ont une double signification. Ils traduisent d'un côté la valeur des certifications à l'intérieur du système éducatif et de formation, en organisant en principe la progression d'un niveau à l'autre (du niveau 5 de la grille française, CAP/BEP, au niveau 4 , bac pro en France par exemple, du niveau 4 - inférieur, au niveau 5 - supérieur, pour deux niveaux de diplômes infirmiers en Hollande...). Mais ils s'organisent aussi en fonction de la valeur externe de la certification sur le marché du travail dans la mesure où ces niveaux sont plus ou moins articulés à une hiérarchie des postes et des fonctions, notamment en servant de référence dans les conventions collectives ou dans les grilles de la fonction publique ( $c f$. Méhaut, 1997 pour cette notion de double valeur des diplômes). Ils ont par ailleurs été bâtis pour partie sur une logique d'inputs (années, durées de formation) et pas d'outcomes. Ainsi pour la France, le CAP de maçon et le titre du ministère de l'Emploi de conducteur de poids lourd sont référencés au même niveau $(\mathrm{V})$ dans le répertoire national des certifications et correspondent en principe à des positions d'ouvrier qualifié. En Hollande, les deux titres son référencés au niveau 2 de la grille nationale. Le cas du diplôme d'infirmier est particulièrement intéressant de ce point de vue. D'une durée de trois ans après le baccalauréat, les études d'infirmière sont pourtant considérées, en France, comme étant du premier niveau du supérieur court (niveau III français). Mais des mouvements de protestations se sont récemment développés autour $\mathrm{du}$ « reclassement » de ce diplôme au niveau « licence professionnelle », mouvements qui vont probablement se poursuivre à mesure de l'articulation plus étroite des formations de santé à l'Université. L'enjeu est ici double: interne - obtenir plus de fluidité « verticale » vers les masters - et externe - le reclassement au niveau A dans les grilles de la fonction publique. En Hollande, les études d'infirmières s'organisent en deux niveaux (4 et 5 dans la grille hollandaise). Le niveau 4 permet l'accès au niveau 5 . Le premier relève plutôt de l'enseignement secondaire (au sens français du terme), le deuxième plutôt de l'enseignement supérieur.

- En Angleterre, toutes les certifications (professionnelles et académiques) sont théoriquement alignées sur huit niveaux dans le Qualification and Credit Framework $(\mathrm{QCF})^{6}$, qui est lui-même étroitement aligné avec les 8 niveaux du CEC. Néanmoins, en pratique, la situation actuelle ne correspond pas aux principes théoriques. Le problème principal est le manque de spécification des savoirs dans le contenu des certifications de type $N V Q s$, qui sont basées sur des outputs en termes de performance, surtout au niveau 2 mais aussi au niveau 3 (Jessup 1990). En pratique, les $N V Q s 2$ et 3, qui sont complètement basées sur une approche "outcomes », n'ont pas une valeur équivalente aux autres certifications plus académiques, ni sur le marché du travail ni sur le marché des études (Oates, 2004). Pour l'infirmier, le diplôme

${ }^{6}$ L'équivalent, en Angleterre, de notre répertoire national des certifications. 
de niveau 5 a une valeur effective dans les deux domaines, même hors du secteur de la santé, à cause de sa position dans l'enseignement supérieur. Il en est de même pour l'informaticien, surtout au niveau de Bachelor (6), mais aussi pour le Higher Apprenticeship (5) et même pour le BTEC National Certificate (3), parce que ces deux derniers sont toujours dans un modèle de type input/output. La mobilité dans et entre les deux marchés est difficile pour les conducteurs et les maçons, parce que leurs $N V Q s$, du type «output», ne sont pas reconnues comme les équivalents académiques du niveau 2 que sont les diplômes d'école (5 sujets de GCSE A-C, examen pour quasiment tous les élèves de seize ans), bien qu'elles soient positionnées comme équivalentes dans le Qualification and Credit Framework. Ici encore, c'est bien la question de la double valeur des certifications qui est en cause.

- En Allemagne, les certifications de maçon et de conducteur de poids lourd relèvent du système dual. À ce titre, elles correspondent en principe au même niveau « homogène » de Facharbeiter (ouvrier qualifié, au sens allemand), au même titre que toutes les autres certifications de ce système. On sait cependant qu'il existe une forte hiérarchie implicite au sein du système dual, qui s'exprime notamment dans la sélection des apprentis par les entreprises. Par ailleurs, ces certifications n'ont pas la même valeur "verticale » que dans les deux premiers pays. Certes, au sein du système dual existent des progressions vers des titres et des emplois de technicien et d'agent de maitrise mais ouvertes seulement après une certaine expérience professionnelle. Par ailleurs, les passerelles avec les autres pans du système de formation (et notamment avec l'enseignement supérieur) sont faibles, voire inexistantes. Le titre d'infirmière relève d'un autre système (celui des écoles professionnelles de la santé). Mais il pourrait être assimilé à un « niveau » de sortie du système dual, ou au niveau 4 Hollandais car, explicitement, il n'est pas considéré comme relevant de l'enseignement «supérieur». Toutefois, là encore, en l'absence de grille nationale, il n'y a pas de positionnement explicite.

La confrontation aux niveaux du CEC, dès lors qu'elle soulève des enjeux forts en termes de hiérarchie sociale ou professionnelle, ne se fera probablement pas sur la seule question des descriptifs de compétences. Par ailleurs, l'une des questions posée par le CEC est celle de l'homogénéité « horizontale » du classement des certifications. On pourrait parfaitement imaginer par exemple que le conducteur de poids lourd «ministère du Travail » en France soit classé au niveau 2 du CEC sur les dimensions savoir et aptitudes, mais au niveau 3 sur la dimension " compétence» (le référentiel met l'accent sur la capacité à "prendre des responsabilités pour effectuer des tâches et à adapter son comportement aux circonstances pour résoudre des problèmes »).

\section{STRATÉGIES NATIONALES, TRANSPOSITION ET RÉTRO-ACTIONS : QUELQUES HYPOTHÈSES}

Au moment où ce texte est écrit, les premières expériences de transposition sont en cours ( $c f$. aussi EQF, 2008, pour une étude comparée sur sept pays). Au vu de l'examen ci-dessus, on se contentera de formuler quelques hypothèses en forme de scénarios nationaux.

- Les concepts du CEC sont à la fois les plus proches... et les plus éloignés du système anglais de $N V Q s$. La classification des $N V Q s$, particulièrement sur la première dimension verticale du CEC (savoirs) est la plus difficile. Mais l'enjeu social est faible car les $N V Q s$ n'ont pas encore vraiment fait système du point de vue de leur valeur interne (progression dans un système de certification et de formation) sauf dans certains secteurs où ce processus s'amorce (cf. Grimshaw, Carrol, 2008, pour l'exemple de la santé). Il est aussi faible en raison de la modeste valeur marchande des $N V Q s$ sur le marché du travail. On peut donc penser que la transposition sera aisée, et se fera de façon «mécanique », chaque niveau de $N V Q$ trouvant sa place à un niveau du CEC. Mais on voit bien ici que se posera alors la question de la confiance mutuelle qu'est censée créer le CEC entre les acteurs des systèmes de certification des différents pays. Est-il concevable, en France par exemple, qu'une $N V Q 3$ soit référencée au même niveau qu'un bac professionnel ? Appartenant en revanche clairement au registre de l'enseignement supérieur, 
le diplôme d'infirmière devrait trouver sa place par l'intermédiaire de la grille «de Bologne», a priori plutôt au niveau 5 du CEC en équivalent de la position du diplôme français (actuellement de niveau III dans la grille française... qui correspondrait au niveau $5 \mathrm{du}$ CEC). Mais ce diplôme permet aussi de progresser en un an jusqu'au bachelor in Nursing (niveau $6 \mathrm{du}$ CEC)... où devrait se retrouver le diplôme français s'il acquiert une équivalence à la licence.

- Les concepts du CEC sont très loin des concepts allemands, tant sur la question des compétences, que sur celle des niveaux. Toutefois, la logique du CEC vient percuter les difficultés actuelles du système dual et plus largement du système de formation: introduire des niveaux, c'est expliciter, éventuellement faciliter des parcours verticaux de l'apprentissage vers d'autres filières d'enseignement; c'est aussi répondre aux critiques qui, sur les données issues des statistiques internationales, situent l'Allemagne en retard en termes d'accès à l'enseignement supérieur alors qu'une partie des formations en apprentissage pourraient relever d'un bac pro (donc d'un niveau IV dans la grille française) voire d'un BTS (brevet de technicien supérieur)/DUT (et donc d'un niveau III). C'est aussi prendre acte de la forte différenciation interne à l'apprentissage, comme des différences entre les diplômes d'apprentissage et les autres diplômes de formation professionnelle. Il y a donc des enjeux politiques forts en termes de politique éducative... mais aussi des freins sociaux forts. Introduire des niveaux explicites au sein de l'apprentissage mettrait en cause l'unité «symbolique» de l'ouvrier qualifié ( $c f$. par exemple les critiques adressées au CEC par Drexel, 2005 ; $c f$. aussi Hanf, Volker, 2008). En effet, en théorie, tous les titulaires d'un diplôme d'apprentissage font partie de la catégorie d'ouvrier qualifié, sans distinction de niveau. L'usage du CEC pourrait être alors soit rejeté soit, au contraire, utilisé de façon très volontariste par les politiques comme un «levier» d'évolution du système. Le CEC serait alors un instrument de changement « organique » du système allemand ${ }^{7}$.

\footnotetext{
${ }^{7} \mathrm{Au}$ moment où ce texte est écrit, une première version de cadre national allemand est testée dans des groupes de travail rassemblant des experts (éducation, partenaires sociaux, recherche...). Une fois cette démarche validée, une décision gouvernementale établira le statut du nouveau cadre national et les conditions de son articulation au CEC.
}

- Le flou des catégories du CEC s'accommode assez facilement du flou des catégories à la française. Il n'y a, a priori, pas trop de difficultés à faire rentrer les savoir, savoir-faire et savoir-être dans les trois dimensions verticales des compétences du CEC. La question des niveaux est par contre plus sensible. Sans toutefois soulever de gros problèmes, si l'on considère que les niveaux sont homogènes horizontalement sur les trois dimensions. Dans ce cas, le DUT informatique trouve naturellement sa place au niveau $5 \mathrm{du}$ CEC, le titre d'infirmière aussi (mais il y aura bataille sociale pour le placer au niveau 6, équivalent d'un bachelor/licence professionnelle). Par un jeu de dominos, le bac professionnel trouvera sa place au niveau 4 (et probablement tous les bac professionnels car les situer à des niveaux différents toucherait au mythe de l'unité de niveau du baccalauréat). En poursuivant l'hypothèse d'une interprétation «à minima » du CEC, à la fois «mécanique » comme à l'anglaise, mais ici pour éviter de toucher aux subtils équilibres sociaux autour de la grille des niveaux français, les CAP et autres titres de l'actuel niveau V français trouveraient tous leur place au niveau $3 \mathrm{du}$ CEC. Si l'on fait éclater, en revanche, l'homogénéité de niveau des trois dimensions des compétences, on pourrait penser que les certifications de niveau 5 français s'échelonnent différemment selon les niveaux 2 et 3 du CEC. Ainsi par exemple, certains CAP formant à des métiers d'art artisanaux pourraient être considérés comme préparant à plus d'autonomie sur la dimension «compétence» du CEC que d'autres CAP industriels. Cette question est par ailleurs, pour la France, articulée avec une réflexion en cours sur la refonte de la grille nationale des niveaux. Là aussi, le CEC pourrait être alors le levier d'une évolution nationale.

- Le cas de la Hollande est aussi sensible. D'une part, les dimensions compétences du CEC ne correspondent pas à celles des référentiels nationaux. D'autre part, la grille hollandaise de niveau a, nous l'avons vu, une forte valeur tant interne au système de formation qu'externe sur le marché du travail. Par ailleurs se pose pour la Hollande (mais aussi pour les autres pays) la question du statut du niveau $1 \mathrm{du}$ CEC. On peut en effet lire ce niveau 1 comme celui des compétences de base d'une fin de scolarité obligatoire. Dans ce cas, le niveau 1 de l'enseignement 
professionnel hollandais correspondrait plus au niveau 2 du CEC... mais alors que faire des quatre autres niveaux clairement hiérarchisés du système hollandais ? Le $5^{\mathrm{e}}$ niveau (que l'on pourrait assimiler à l'enseignement supérieur court en France) trouverait sa place au niveau $6 \mathrm{du}$ CEC. Mais quid alors des diplômes de l'enseignement supérieur? Ou alors lire le niveau 1 du CEC comme le premier niveau d'enseignement professionnel correspondant au niveau 1 hollandais. Dans ce cas, la transposition serait simple, mécanique, sans toucher aux équilibres nationaux. Mais la référence aux concepts et descripteurs du CEC serait purement formelle ${ }^{8}$.

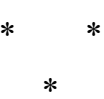

Les scénarios ci-dessus sont, à ce stade, très spéculatifs. Ils n'intègrent pas assez l'hétérogénéité des systèmes nationaux de certification et les stratégies éventuellement différentes des acteurs porteurs de telle ou telle certification, tout particulièrement pour la Grande-Bretagne et la France. Ils n'intègrent pas non plus les éventuelles rétro-actions, dans un processus d'apprentissage mutuel autour du CEC. Ils soulignent cependant les tensions probables, inhérentes à un processus complexe :

- d'une part du fait des différentes conceptions des compétences, plus ou moins intégratives selon les pays, qui viennent rencontrer le flou des concepts du CEC ;

- d'autre part du fait de la logique purement « outcomes » du CEC qui, même si elle correspond à une tendance d'évolution de tous les systèmes nationaux (dont la VAE - validation des acquis de l'expérience - est la manifestation la plus récente en France), va cependant se heurter à des systèmes qui, y compris en Angleterre, restent plus mixtes, mélan-

\footnotetext{
${ }^{8} \mathrm{Au}$ moment où ce texte est écrit, les discussions autour du CEC sont très peu avancées en Hollande, et les acteurs du système national de formation professionnelle peu pressés d'entrer dans ce débat. Une exception notable vient des producteurs de certifications «privées » qui verraient bien leurs certifications « homologuées » dans le cadre du CEC. Un cadre national doit être construit entre 2009 et fin 2010. Les premiers débats montrent une volonté de prendre de la distance par rapport au CEC, avec des descripteurs « nationaux » et un nombre de niveaux à déterminer indépendamment de ceux du CEC.
}

geant les logiques d'outcomes et d'input (c'est-à-dire les «compétences» produites et la façon de les produire).

Dans ces conditions, le CEC permettra-t-il de créer des zones de confiance mutuelle entre acteurs des systèmes de formation, nécessaires à ses objectifs, notamment pour ce qui est de la mobilité des élèves et étudiants? Probablement oui pour certains diplômes répondant à des logiques très professionnelles. Plus difficilement pour d'autres :

- Là ou existe déjà une norme européenne, comme pour le diplôme d'infirmier, cela semble possible. Mais le CEC pourrait aussi révéler l'ambiguïté de ces normes et mettre en évidence des décalages.

- Là où les proximités entre systèmes sont fortes (dominance de l'apprentissage comme en Allemagne, Autriche, hiérarchie "académique » des diplômes comme en France, en Italie) et, dans la mesure où une autorité nationale clairement reconnue assurera le contrôle de la transposition, cette confiance pourrait se développer, au moins entre pays ayant des proximités systémiques.

- On peut aussi penser que les conditions de fonctionnement de marchés du travail transnationaux (notamment pour les multinationales à recrutement européen, qu'il s'agisse par exemple aujourd'hui de l'informatique, de la banque, des transports internationaux...) joueront un rôle homogénéisateur. Mais cette hypothèse, plausible dans le cas d'un diplôme ayant seulement valeur sur le marché du travail, l'est moins dans les situations de double valeur où les enjeux internes aux systèmes de formation (et entre organismes formateurs éventuellement concurrents) joueront pleinement.

Au-delà de ces difficultés immédiates, et des enjeux forts qu'il révèle, le Cadre européen des certifications témoigne aussi des limites de la politique européenne. Sans compétence explicite dans le domaine de la formation, essayant d'en construire une dans celui de la certification, l'Union a un faible pouvoir de régulation. Le futur du cadre européen repose donc plus sur la qualité du dialogue entre pays qu'il peut susciter. Mais l'outil peut-il suffire à la politique? 
Bessy C. (2000), « La certification des compétences en Grande-Bretagne. Les risques d'exclusion induits par la valorisation d'aptitudes générales ", Formation Emploi, n 71 , pp. 21-35.

Bjornavold J., Coles M. (2008), « Gérer l'éducation et la formation: l'exemple des cadres de certifications ", Revue européenne de formation professionnelle, ${ }^{\circ}$ 42-43, pp. 227-265.

Bosch G., Charest J. (2009), "Vocational training systems in ten countries and the influence of the social partners" paper presented to the IIRA $14^{\text {th }}$ World Congress, Lima, Peru, 11-14 September.

Bosch G. et Charest J. (à paraître en 2010), "Vocational training : international perspectives", in Bosch, Charest (Eds) Vocational Training, International Perspectives, Routledge, London, pp. 1-26.

Bosch G. (à paraitre en 2010), "The revitalisation of the Dual Sytem of Vocational Training in Germany" in Bosch, Charest (Eds) Vocational Training, International Perspectives, Routledge, London, pp. 136-161.

Bouder A., Kirsch J.-L., (2008), "Drawing up European competence standards, Some thoughts about the experience gained in France", Training and Employment, $\mathrm{n}^{\circ} 78$.

Brockmann M., Clarke L., Winch C. (2008), "Knowledge, Skills, Competence: European divergences in vocational education and training (VET) the English, German and Dutch cases", Oxford Review of Education.

Brockmann M., Clarke, L. Winch C. (2009), Cross national Equivalence of Vocational Skills and Qualifications: synthesis report, The case of England

Clarke L., Winch C. (2007), Vocational Education: International Approaches, Developments and Systems, Oxford, Routledge.
Brockmann M., Clarke L., Méhaut Ph., Winch C. (2008), "Competence based-Vocational Education and Training (VET): the Cases of England and France in a European perspective", Vocation and Learning, vol. 1, n 3, pp. 227-244.

Coles M., Oates T. (2004), European Reference Levels for Education and Training an important parameter for promoting credit transfer and mutual trust. Final Report. London, Qualifications and Curriculum Authority.

Drexel I. (2005), Das Duale System und Europa : ein Gutachen im Aufrag von Ver.di und IG-Metall. Berlin.

EQF Implementation: towards a network of National Coordination Points (2008), report of the EQF Network Testing Project, Leonardo, www.eqfnettesting.eu

Felstead A. (1998), Output-related funding in vocational education and training, a discussion paper and case studies, Cedefop, Thessaloniki.

Gehin J.-P., Méhaut Ph. (1993), Apprentissage ou formation continue? Stratégies éducatives des entreprises en France et en Allemagne, Paris, L'Harmattan.

Greinert W.D. (2005), «Les systèmes européens de formation professionnelle, réflexion sur le contexte théorique de leur évolution historique », Formation Professionnelle, $\mathrm{n}^{\circ}$ 32, pp. 19-27.

Grimshaw D, Carroll M. (2008), "Improving the position of low wage workers through new coordinating institutions : the case of public hospitals" in Lloyd C., Mason G., Mayhew K. (Eds), Low wage work in the United Kingdom, Russel sage, New York.

Hanf G., Volker R. (2008), « Cadre européen et cadre national des certifications - Un défi pour la formation professionnelle en Allemagne », Revue européenne de Formation Professionnelle, pp. 125-142. 
Hanf G. (2009), Quick Scan Report on German VET, Unpublished report for Nuffield Foundation project.

Jessup G. (1991), Outcomes: NVQs and the Emerging Model of Education and Training. London: Falmer.

Laffan B., Shaw C. (2005), Classifying and Mapping the OMC in different policy area, Newgov, www.eunewgov.org

Maillard F (sous la dir.) (2008), Des diplômes aux certifications professionnelles, nouvelles normes et nouveaux enjeux, Rennes, Presses Universitaires.

Markowitsch J., Luomi-Messerer K. (2008), «Développement et interprétation des descripteurs du cadre européen des certifications », Revue européenne de Formation Professionnelle, $\mathrm{n}^{\circ}$ 42-43, pp. 38-65.

Méhaut Ph. (1997), «Le diplôme, une norme multivalente?» in Möbus, Verdier (Eds.), Les diplômes professionnels en France et en Allemagne, conceptions et jeux d'acteurs, Paris, L'Harmattan, pp. 263-273.

Méhaut Ph. (2009), Quick Scan Report on France, Unpublished report for Nuffield Foundation project.
Möbus M., Verdier E. (1997), Les diplômes professionnels en Allemagne et en France, conception et jeux d'acteurs, L'Harmattan, Paris.

Morvan Y (sous la dir.) (2007), La formation tout au long de la vie, Nouvelles questions, nouvelles perspectives, PUR, Rennes.

Oates T. (2004), "The Role of Outcomes-Based National Qualifications in the Development of an Effective Vocational Education and Training System; the case of England and Wales", Policy Futures in Education, 2(1), pp. 53-71.

Sellin B. (2008), « La proposition d'un cadre européen des certifications : possibilité et limites de sa transposition dans la réalité », Revue européenne de Formation Professionnelle, ${ }^{\circ}{ }^{\circ} 42-43$, pp. 4-21.

Verdier E. (2008), «L'éducation et la formation tout au long de la vie : une orientation européenne, des régimes d'action publique et des modèles nationaux en évolution », Education et Société, 40, pp. 195-225.

Vinokur A. (1995), «Réflexions sur l'économie du diplôme », Formation Emploi, n 52 , pp 151-183.

Westerhuis A. (2009), Quick Scan Report on the Netherlands, Unpublished report for Nuffield Foundation project.

\section{Résumé}

Le cadre européen des certifications : quelles stratégies nationales d'adaptation? Philippe Méhaut et Christopher Winch

La coordination européenne des politiques de formation professionnelle s'affirme progressivement. Qu'i s'agisse des indicateurs d'objectifs partagés et comparés, des "crédits européens de formation professionnelle », de la mise en place d'un Cadre européen des certifications, ces outils de politique publique européenne interpellent les systèmes nationaux de certification. L'examen, dans quatre pays, de la structure des certifications et des concepts qui les sous-tendent, montre leur diversité tant entre pays qu'au sein d'un même pays. Certaines conceptions sont difficilement compatibles avec celles du cadre européen. On peut alors formuler quelques hypothèses sur les stratégies nationales de transposition de ces certifications dans le nouveau cadre européen.

Mots clés

Certification, politique européenne, politique de la formation, comparaison internationale Journal of Economic Literature: I 21 